Cahiers d'études africaines

\title{
MÜLLER, Tanja R. - Legacies of Socialist Solidarity. East Germany in Mozambique
}

Constantin Katsakioris

\section{OpenEdition}

Journals

Édition électronique

URL : http://journals.openedition.org/etudesafricaines/20779

DOI : $10.4000 /$ etudesafricaines. 20779

ISSN : 1777-5353

\section{Éditeur}

Éditions de l'EHESS

\section{Édition imprimée}

Date de publication : 1 juillet 2017

Pagination : 469-471

ISBN : 978-2-7132-2688-5

ISSN : 0008-0055

\section{Référence électronique}

Constantin Katsakioris, « MülleR, Tanja R. - Legacies of Socialist Solidarity. East Germany in

Mozambique », Cahiers d'études africaines [En ligne], 226 | 2017, mis en ligne le 01 avril 2017, consulté le 23 septembre 2020. URL : http://journals.openedition.org/etudesafricaines/20779 ; DOI : https:// doi.org/10.4000/etudesafricaines.20779

Ce document a été généré automatiquement le 23 septembre 2020.

(c) Cahiers d'Études africaines 


\title{
MÜLLER, Tanja R. - Legacies of Socialist Solidarity. East Germany in Mozambique
}

\author{
Constantin Katsakioris
}

\section{RÉFÉRENCE}

MüLLER, Tanja R. - Legacies of Socialist Solidarity. East Germany in Mozambique. Lanham, Lexington Books, 2014, 205 p., bibl.

1 C'est à l'histoire et aux trajectoires d'élèves, alors âgés de 10 à 14 ans, venus en 1982 de la République populaire du Mozambique pour étudier à l'École de l'amitié, Schule der Freundschaft, une école technique secondaire qui venait d'être ouverte à Stassfurt, en République démocratique allemande (RDA) que Tanja R. Müller consacre cet ouvrage. Inspiré par l'exemple des écoles internationales ouvertes sur l'Isla de la Juventud à Cuba, cette école-internat assez originale où les élèves passaient six ans, avait été créée à la suite d'un accord entre le Front révolutionnaire de libération du Mozambique (FRELIMO) et la RDA. Elle avait pour objectif de préparer de futurs cadres techniques moyens, hommes et femmes, qui travailleraient dans des entreprises publiques mises en place par la coopération avec la RDA; en même temps, elle devait participer à la formation de l'homem novo, l'homme nouveau, progressiste et patriote, dont la contribution était attendue dans la construction d'une société socialiste au Mozambique indépendant.

2 Anthropologue et spécialiste des questions d'enseignement et de développement, Tanja Müller se concentre dans son livre sur l'héritage (legacies), ou ce qui en reste, de cette coopération éducative qui à l'époque portait l'empreinte idéologique de la Guerre froide. Elle l'étudie à partir d'entretiens biographiques réalisés en allemand et en anglais au Mozambique en 2008 auprès d'un échantillon de 35 des 899 anciens élèves, hommes et femmes, arrivés en 1982 en RDA. Nourrie aussi par des discussions 
informelles aussi bien avec eux qu'avec leurs camarades de classe, cette étude est complétée, à un moindre degré, par des recherches dans les archives de l'ancienne RDA. Ce faisant, l'ouvrage soulève des questions qui dépassent de loin celle de l'héritage de l'École de l'amitié. Dans un premier temps, T. Müller nous convie à un aperçu des engagements de la RDA en Afrique subsaharienne avant de se focaliser sur la coopération particulière développée avec le Mozambique, pour y situer ensuite l'École de l'amitié en dressant le tableau politique, éducatif et administratif de cette coopération. Concis et bien écrit, le premier chapitre fait état des prémisses et des objectifs de l'alliance entre les deux pays et met l'accent sur le rôle central de leur coopération éducative. Dans le deuxième chapitre, l'auteure examine les profils sociaux, politiques et ethniques des familles des élèves et enquête sur leurs aspirations et appréhensions avant le départ. Originaires de diverses régions et parlant des langues différentes, la plupart des élèves étaient, souligne-t-elle, issus du milieu rural, autant de conditions qui ont marqué le déracinement de ces enfants. Toutefois, comme le montre le chapitre 3 qui revisite le vécu allemand des élèves, leur adaptation en RDA était plutôt excellente, et les interviewés ont jugé fort agréables les conditions de vie qui leur avaient été offertes. Sur la foi des témoignages, Tanja Müller parvient à élaborer ce que l'on peut appeler - et qu'elle mentionne du reste comme - les «mémoires d'un paradis ».

3 Deux événements néanmoins viennent structurer ces mémoires en leur servant de marqueurs. Le premier est la mort de Samora Machel. Survenue en octobre 1986, la disparition du premier Président mozambicain constitue, dans tous les entretiens, le point de départ du "récit de la trahison », car elle est interprétée, sinon vécue, comme la cause de l'abandon du socialisme par les successeurs de Machel. Le second est l'assassinat de leur camarade de classe Carlos Conceição en septembre 1987 par des jeunes Allemands. Cet événement est évoqué comme le début de l'ère des skinheads et structure le « récit du racisme » qui, selon la plupart des récits des interviewés, n'a pas été suffisamment reconnu et pris en compte.

4 À leur retour au Mozambique post-Machel en 1988, les anciens élèves qui avaient quitté un pays à option socialiste se trouvent soudain confrontés à une situation où ce même pays est embarqué dans des réformes économiques, dictées par les marchés et les bailleurs de fonds internationaux et dont le coût social s'avère exorbitant. Dans ces conditions, les anciens élèves de l'École de l'amitié dont le diplôme allemand n'était pas reconnu comme un baccalauréat, n'avaient - comme le montre le suivi de leurs trajectoires réalisé dans le chapitre 4 - aucune place. Ils ont d'ailleurs été contraints de refaire deux années de lycée au Mozambique, avant de s'engager pour nombre d'entre eux dans l'armée, et dans une sanglante guerre civile contre la Résistance nationale mozambicaine (RENAMO). Ce sont leurs difficultés et désillusions une fois de retour dans le pays qui renforcent les mémoires du "paradis allemand» et le sentiment d'avoir été trahis par un gouvernement désormais néolibéral en place. Ce sentiment conduit l'auteure à retracer, dans les chapitres 4 et 5, ainsi que dans la conclusion, l'héritage des années allemandes. Parmi les trente-cinq personnes interviewées, seules trois pratiquent des métiers en lien avec la spécialisation obtenue en Allemagne, preuve que l'objectif principal de l'École de l'amitié n'a pas été atteint. Toutefois, au moins dix-neuf anciens élèves, qui ont combattu dans les rangs de l'armée et ont dû plusieurs fois se réorienter, considèrent avoir réussi sur le plan professionnel et social, attribuant leur réussite à leur éducation socialiste et allemande. L'auteure en déduit que, si leur formation en RDA n'a pas servi la cause du socialisme et de sa construction 
au Mozambique, elle a néanmoins été d'une grande utilité pour la majorité des anciens élèves.

Héritage personnel autant que collectif, l'expérience allemande des élèves de la Schule der Freundschaft est, à ce titre, d'une double importance. Pour T. Müller, cet héritage est avant tout professionnel et socioculturel, mais aussi socialiste et allemand. Socialiste, parce que les anciens élèves n'ont pas rompu avec les idéaux du socialisme auxquels ils croient encore fortement et qui leur a permis de se constituer en groupe d'entraide. Certains se sont engagés sur le terrain municipal, au niveau local, même si aucun d'eux n'est impliqué dans la vie politique nationale. Allemand, car ils aiment planifier leur vie et se réclament de cet héritage particulier de l'éducation et de la rigueur allemandes. Socioculturel aussi car, ainsi que l'observe l'auteure, tous les anciens élèves vouent un culte à l'éducation; les couples d'anciens élèves ont moins d'enfants et essaient de leur payer de bonnes écoles, les femmes sont émancipées et les relations conjugales bien différentes de celles de couples mozambicains traditionnels. Sur le plan professionnel enfin, plusieurs anciens élèves ont mis en valeur leur expérience internationale pour obtenir des contrats dans les organisations non gouvernementales. T. Müller démontre ainsi concrètement l'impact significatif de l'éducation en Allemagne sur la vie de ses interlocuteurs.

Bien qu'elle ne soit pas directement annoncée et qu'elle soit peu développée, l'étude historique existe cependant de fait dans ce livre. Abordée uniquement par le biais d'un détour par l'histoire orale, celle-ci dessert un travail qui, pour servir d'arrière-plan à la compréhension de l'École de l'amitié et de son héritage, aurait mérité des analyses plus systématiques et approfondies, notamment en ce qui concerne certaines expériences ou questions soulevées. Ainsi, apprenons-nous que les élèves mozambicains portaient des vêtements occidentaux - ce que les Allemands de l'Est leur enviaient souvent mais nous ne savons pas comment ils se les procuraient. De même, l'auteure mentionne plusieurs fois que les élèves passaient souvent les week-ends ou les vacances dans des familles allemandes; cependant aucune information sur les relations avec la société d'accueil et leur impact ne vient étayer ces propos. Ceci trahit le goût d'inachevé que l'on peut ressentir et déçoit par moments les attentes du lecteur. Il n'empêche que l'ouvrage est une collecte inestimable d'expériences de ces anciens élèves de l'École de l'amitié, tous adolescents ou pré-adolescents à leur arrivée en Allemagne de l'Est, dont les trajectoires à leur retour au pays nous apprennent beaucoup sur le devenir de certains États africains qui nourrissaient un projet social par la suite laminé en raison du tournant pris par l'histoire depuis la chute du mur de Berlin. 